\title{
The relationship between neck angles and ground reaction forces in schoolchildren during backpack carriage
}

\author{
Dalia Mohammed Mosaad ${ }^{1,2}$, Amr Almaz Abdel-aziem $^{3}$ \\ ${ }^{1}$ Department of Basic Science, Faculty of Physical Therapy, Cairo University, Giza, Egypt; ${ }^{2}$ Department of Basic Science, \\ Faculty of Physical Therapy, Badr University, Cairo, Egypt; ${ }^{3}$ Department of Biomechanics, Faculty of Physical Therapy, \\ Cairo University, Giza, Egypt
}

\section{Summary}

Study aim: This study aimed to examine the effect of carrying backpacks on neck posture and ground reaction forces (GRFs) and to investigate the relationship between neck angles and GRFs during backpack carriage in schoolchildren.

Material and methods: The craniohorizontal angle (CHA), craniovertebral angle (CVA), sagittal shoulder posture (SSP) and GRFs were measured in right-handed schoolchildren (14 male and 12 female) with mean age $10.17 \pm 1.15$ years during loaded and unloading conditions. The Qualisys motion analysis system with a force plate was used to assess the neck angles and GRFs.

Results: During backpack carriage there was a significant increase in the CHA $(\mathrm{p}=0.001)$, significant decrease in the CVA and SSP ( $\mathrm{p}=0.001,0.016$ respectively), no significant difference in the normalized (scaled to body weight) vertical GRFs $(\mathrm{p}>0.05)$, and a significant increase in the anterior braking and posterior propulsive GRFs $(\mathrm{p}=0.035,0.002$ respectively) compared to the unloading condition. While carrying a backpack there was a moderate negative correlation between the SSP and first vertical GRF $(\mathrm{r}=-0.464)$ and a strong negative correlation with the second vertical GRF $(\mathrm{r}=-0.571)$ and the posterior propulsive GRF ( $\mathrm{r}=-0.587)$.

Conclusion: Carrying a backpack weighing $15 \%$ of the child's body weight changes the head posture and increases the normalized value of the anterior-posterior shear force. During backpack carriage, decreasing the SSP is associated with increasing the load acceptance, thrusting and posterior propulsive forces. Increasing the shearing force may lead to development of postural abnormities. Consequently, the ideal backpack weight should be considered by parents and teachers.

\section{Key words: Schoolchildren - Backpack - Neck posture - Ground reaction force - Load carriage}

\section{Introduction}

Backpack use amongst schoolchildren has become the most popular means of transporting luggage to and from school $[6,17]$. Carrying a backpack leads to postural changes which require many adjustments of the trunk and limbs to accommodate to the new stresses, and maintaining upright posture and equilibrium [29].

The prevalence of musculoskeletal symptoms due to backpack carriage is higher in younger age groups than in their older counterparts [41]. A heavy bag pack has been considered as a risk factor for developing musculoskeletal pain among children in the age range 9-16 years [49]. The backpack weight of school students has been reported to range from $10 \%$ to as high as $25 \%$ of their body weight
(BW) and may have a harmful impact on their body [33]. However, half of the schoolchildren carry schoolbags weighing more than $10 \%$ of $\mathrm{BW}$, which is more frequently associated with tiredness and back pain [20]. Furthermore, the recent review study of Janakiraman et al. [21] stated that the backpack load limit for schoolchildren should be $10-15 \%$ of BW. Excessive loading on the back can lead to forward bending of the trunk and neck that may be the biomechanical cause of musculoskeletal pain [41].

Inappropriate use of backpacks is not healthy for anyone, especially for children, who are more susceptible to injury because their bodies are growing and developing [37]. Carrying a backpack weighing $10 \%$ of BW leads to a reduction in gait velocity and cadence, an increased double support time and trunk forward lean, which could be a compensatory mechanism to minimize either the 
induced gait instability or mechanical strain on the musculoskeletal system [44]. Many studies conducted on adults have indicated that load carriage changed the gait kinematics $[5,26,43]$ and ground reaction forces (GRFs) $[4,5,8,32,43]$. A recent study conducted on primary school students found that the heavier the school backpack was, the higher was the pressure and force distribution under different foot regions [1]. Moreover, carrying school backpacks with loads of more than $10 \% \mathrm{BW}$ causes changes in the gait kinematics [23].

The measurement of GRFs is an accurate technique for assessing the gait biomechanics. The vertical GRF is related to joint contact forces, which play an important role in the development of musculoskeletal pathological conditions such as low back pain and osteoarthritis [34]. The anteriorposterior GRF is the main component that indicates shear stress [12]. This force represents the friction between the foot and shoe or shoe and ground, and its increase may lead to foot injuries [39] and a tendency to slip [12]. Both of the mentioned GRF components inform about the overall forces acting on the human body [10]. The vertical GRF increased almost three times in student carrying school bags weighing $20 \%$ of BW compared to $10 \%$ of BW [34]. Moreover, it has been reported that carrying a backpack weighing $7.5 \%$ of BW in schoolchildren leads to an increase in GRFs and a more forward head position [29].

Thus, carrying backpacks leads to biomechanical changes that could be the cause of low back pain [45], muscle soreness [22], joint problems [3] and adverse consequences for foot structure and function [31]. Consequently, health care professionals, teachers, and parents should be aware of the need to restrict the backpack weight to the minimum values to solve most of the problems related to the weight of the bag in relation to BW [36]. Therefore, students, staff, and families need to be educated about backpacks' contribution to pain and musculoskeletal complains [37].

To achieve a more comprehensive understanding of the forces acting on the musculoskeletal system, combining the GRFs and neck posture appears to be relevant. However, the relationship between the neck angles and GRFs during backpack carriage has received almost no attention in the academic literature related to children. Therefore, this study aimed to examine the effect of carrying backpacks on neck posture and GRFs and to investigate the relationship between neck angles and GRFs in children during backpack carriage.

\section{Materials and methods}

\section{Participants}

A convenient sample of primary school students was recruited by announcing recruitment criteria through an advertisement in two primary schools in Giza. The volunteers were enrolled after an interview and informing them of the required details of the study. A total of 38 children were interviewed but only 26 met the inclusion criteria and were enrolled in the study (14 male and $12 \mathrm{fe}-$ male), with mean age $10.17 \pm 1.15$ years, mean weight $33.79 \pm 5.72 \mathrm{~kg}$, and mean height $138.28 \pm 7.98 \mathrm{~cm}$. The parents signed a consent form authorizing the child's participation. The study was performed in accordance with the Declaration of Helsinki and approved by the local institutional ethics board. The sample size calculation was conducted using G Power 3.1 software, with a one tailed comparison of difference between two dependent means. The sample size was determined as 26 participants according to $\alpha=0.05$, power $=0.80$ and effect size $=0.50$.

The children were included if they were healthy, physically active and could follow the researcher's instructions. The children were excluded if they had any history of back or neck pain, musculoskeletal disorders or neurological diseases or were obese as defined by a standard definition for child overweight and obesity worldwide [16]. The cut-off point of the mean body mass index (BMI) for overweight and obese children between the ages of 8.5 and 12 years is $18.76-21.22$ for boys and 18.69-21.68 for girls. In this study, the BMI of the children ranged from 13.20 to 20.73 and the mean BMI was $17.61 \pm 2.14$.

Each child had two test captures, one without a bag and the other while carrying a backpack. The order of tests was determined randomly, and was done through a coin toss to determine which test to start with.

\section{Instruments}

For calculating BMI of each participant, the researcher used a digital weight scale and stadiometer. The dimensions of the backpack used by all participants were $37 \times 25 \times 15 \mathrm{~cm}$, which was the backpack style used commonly by schoolchildren. Cervical angles were measured by a 3D motion analysis system, QUALISYS, Sweden. It consists of a camera system (3 Pro Reflex infrared cameras), silver colored reflective markers, and four adhesive reflective markers that were attached to each participant's anatomical landmarks (i.e., the tragus, acromial process, spinous process $(\mathrm{C} 7)$ to calculate the craniohorizontal angle (CHA), craniovertebral angle (CVA), and sagittal shoulder posture (SSP).

The GRFs were measured through a camera system consisting of 3 Pro Reflex infrared cameras. Seven adhesive reflective markers were attached to each participant's anatomical landmarks: at the point between the 2 nd and 3rd metatarsal heads of the right foot, lateral malleolus of the right foot, posterior of the heel of the right foot, lateral joint line of the right knee, over the tibial tuberosity of the right knee, on the upper border of the right patella, and the greater trochanter of the right hip [12]. An AMTI 
(Advanced Mechanical Technology Inc., USA) force plate with sampling rate $120 \mathrm{~Hz}$ was used to measure the antero-posterior and vertical components of the GRFs.

\section{Procedure}

The static test was used to measure the neck angles with and without a backpack that weighs $15 \%$ of BW with mean weight $5.07 \pm 0.86 \mathrm{~kg}$. The backpack straps were adjusted to be carried with its top parallel to the shoulder line [34]. The markers were placed by the same researcher for each participant. Measurements were conducted under the same conditions: the same room temperature. The 3 dimensional motion analysis system was calibrated at the start of each measure to validate the results. The test started with the participant standing in the center of the walkway with bare feet and looking directly ahead, with arms beside their body and evenly distributing their BW on both feet. Then a capture of 4 seconds was recorded by the Q-trac software. The CHA formed at the intersection of the horizontal line and the line joining the tragus of the ear and external canthus of the eye line (1) (Fig. 1) [47]. The CVA formed at the intersection of a horizontal line through the spinous process of C7 and the line of the tragus of the ear line (2) [13], and the SSP formed at the intersection of a horizontal line and the line between the posterior aspect of the acromion process and the C7 line (3) [47].

The dynamic test was used to measure the normalized (scaled to body weight) GRFs: Fv1; the first peak of the

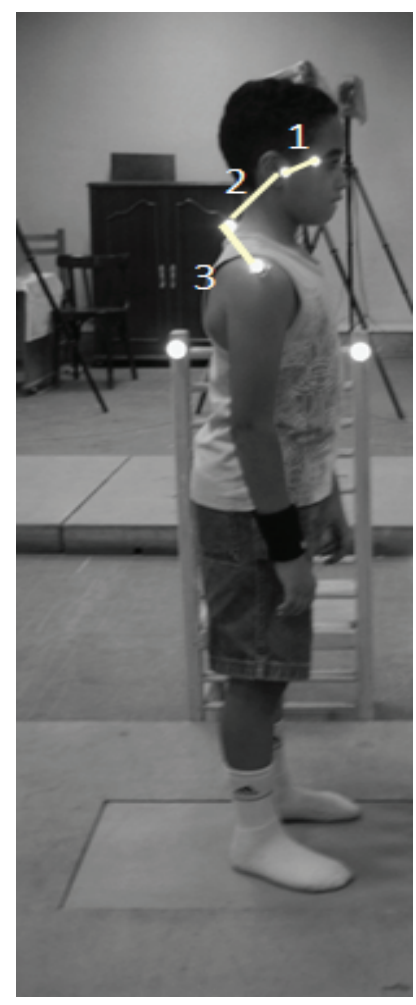

Fig. 1. Representation of lines forming cervical angles vertical force (load acceptance peak), Fv2; the valley of the vertical force (minimum value of the vertical GRF lies between Fv1 and Fv3), Fv3; the second peak of the vertical force (thrusting peak), Fap1: the anterior braking force; the negative value of the anterior-posterior GRF, and lastly Fap2: the posterior propulsive force; the positive value of the anterior-posterior GRF.

Before testing, each child was allowed to practice walking three times to become familiar with the procedure. All participants were asked to walk at their own speed. The children walked a distance of three steps before and three steps after the force plate at their comfortable walking speed [47]. Then, the capture was recorded with and without a backpack. The dynamic test was analyzed by the QUALISYS gait software to determine the GRF components.

\section{Statistical analysis}

All statistical measures were analyzed using SPSS (Armonk, NY: IBM Corp.) version 20.0. Data were screened for the normality assumption and presence of outliers. Descriptive analysis of the participants' demographic data and study-dependent outcome measures (cervical angles and ground reaction forces) was performed. The paired samples t-test was used to compare neck angles and GRFs in children with and without a backpack. Pearson's product moment correlations were used to examine the relationships between neck angles and GRFs. An a priori alpha level of 0.05 was used for all tests.

\section{Results}

Descriptive statistics of the neck angles in children with and without backpack are presented in Table 1 . There was a significant increase in the CHA and decrease in the CVA and SSP $(p<0.05)$ during backpack carriage compared to the unloaded condition.

There was no significant difference in the normalized vertical GRFs (Fv1, Fv2, Fv3) between loading and unloading conditions $(p>0.05)$. There was a significant increase in the normalized anterior braking force (Fap1) and

Table 1. Neck angles (degrees) with and without backpack

\begin{tabular}{lccc}
\hline & Without backpack & With backpack & $p$ value \\
\hline CHA & $22.06 \pm 7.12$ & $29.54 \pm 4.02$ & 0.001 \\
CVA & $49.21 \pm 6.80$ & $44.48 \pm 3.78$ & 0.014 \\
SSP & $25.38 \pm 5.78$ & $23.60 \pm 3.42$ & 0.016 \\
\hline
\end{tabular}

Data are presented as mean (standard deviation), CHA - craniohorizontal angle, CVA - craniovertebral angle, SSP - sagittal shoulder posture. 
Table 2. Normalized ground reaction forces $(\mathrm{N})$ with and without backpack

\begin{tabular}{lccc}
\hline & Without backpack & With backpack & $p$ value \\
\hline Vertical force (Fv1) & $1.02 \pm 0.21$ & $1.03 \pm 0.18$ & 0.337 \\
Vertical force (Fv2) & $0.78 \pm 0.14$ & $0.78 \pm 0.16$ & 0.798 \\
Vertical force (Fv3) & $1.05 \pm 0.21$ & $1.05 \pm 0.19$ & 0.975 \\
For-aft forces (Fap1) & $0.14 \pm 0.06$ & $0.16 \pm 0.04$ & 0.035 \\
For-aft forces (Fap2) & $0.18 \pm 0.04$ & $0.20 \pm 0.04$ & 0.002 \\
\hline
\end{tabular}

Data are presented as mean (standard deviation).

posterior propulsive force (Fap2) during backpack carriage compared to the unloaded condition $(p<0.05)$, as shown in Table 2.

There was no correlation between the CHA and CVA and the normalized Fv1, Fv2, Fv3, Fap1, and Fap2 GRFs with and without a backpack $(p>0.05)$. However, during backpack carriage there was moderate positive correlation between the CHA and Fv1. There was a strong to very strong negative correlation between the SSP and the normalized Fv1, Fv2 and Fv3 $(p<0.05)$, and no correlation with Fap1 and Fap2 $(p>0.05)$ during the unloading condition. However, during backpack carriage, there was a moderate negative correlation between the SSP and the normalized Fv1 $(p<0.05)$, a strong negative correlation with Fv3 and Fap2 $(p<0.05)$, and no correlation with Fv1 and Fap1 $(p>0.05)$, as shown in correlation matrix 1 .

\section{Discussion}

The results revealed that during backpack carriage there was an increase in the CHA and decrease in the CVA and SSP, which agreed with the recent finding that the CHA was greater, and the CVA and SSP were lesser during backpack carriage compared to no load [30]. Moreover, carriage of a lightweight backpack, 7.5\% of BW, resulted in a significant increase in the CHA and decrease in the CVA [29]. The reduction of the CVA and increase of the CHA were consistent with the findings of Vaghela et al. [48], who reported that there was a significant reduction in the CVA (or increased forward head position), and an increase in CHA while carrying a backpack weighing $18 \%$ of $\mathrm{BW}$.

The current changes in the neck angles indicated that the participants extended their neck during backpack carriage as a protective reaction to preserve their normal posture and balance. However, the results of Mo et al. [28] revealed a significant decrease in the $\mathrm{CHA}$ during loading conditions compared to unloading, and another study reported that there was no significant decrease in CHA during loading compared to unloading [13]. This contradiction may be attributed to the usage of different measurement technique. A smaller CHA indicates that the head is tilted more inferiorly and the upper cervical spine is relatively more flexed [13, 38].

The significant decrease in CVA while carrying a backpack is consistent with the findings of Brackley et al. [6], who found a significant changes in children's trunk forward lean and the CVA when the backpack weighs $15 \%$ of BW. Moreover, the children were more likely to have forward head posture and rounded shoulder posture while carrying the same backpack weight, which is corroborated by the current findings [28]. A smaller SSP indicates that the shoulder is relatively oriented forward with respect to $\mathrm{C} 7$ and represents a more rounded shoulder posture $[13,28]$.

The flexed neck posture during backpack carriage may be explained by the high backpack position adopted in the present study [34], while Chen and $\mathrm{Mu}$ [14] reported that carrying the backpack at the level of the 7 th thoracic vertebra leads to severe neck and shoulder discomfort; this high backpack position also caused more head flexion than the other two positions (12th thoracic vertebra and 3rd lumbar vertebra). Moreover, they suggested that carrying a school backpack weighing $15 \%$ of BW should be avoided.

The lack of significant difference in the normalized GRFs while carrying a backpack is not corroborated by the findings of the previous studies [12, 15, 24], which found an increase in the GRFs with increasing backpack weight. Moreover, there is a proportional increase in the vertical GRFs with increasing backpack weight in adult participants [25], while our participants were children. This conclusion depended on the absolute GRFs, not the normalized GRFs, which may be the cause of the inconsistency. The increased values of vertical and anteroposterior GRFs are attributed to the changes in the vertical and horizontal position of the body's centre of mass, caused by the restriction of natural arm swing patterns in children [18].

However, Alghadier [2] concluded that carrying a backpack significantly altered the dynamic foot-loading patterns (peak plantar pressure, peak plantar force and 


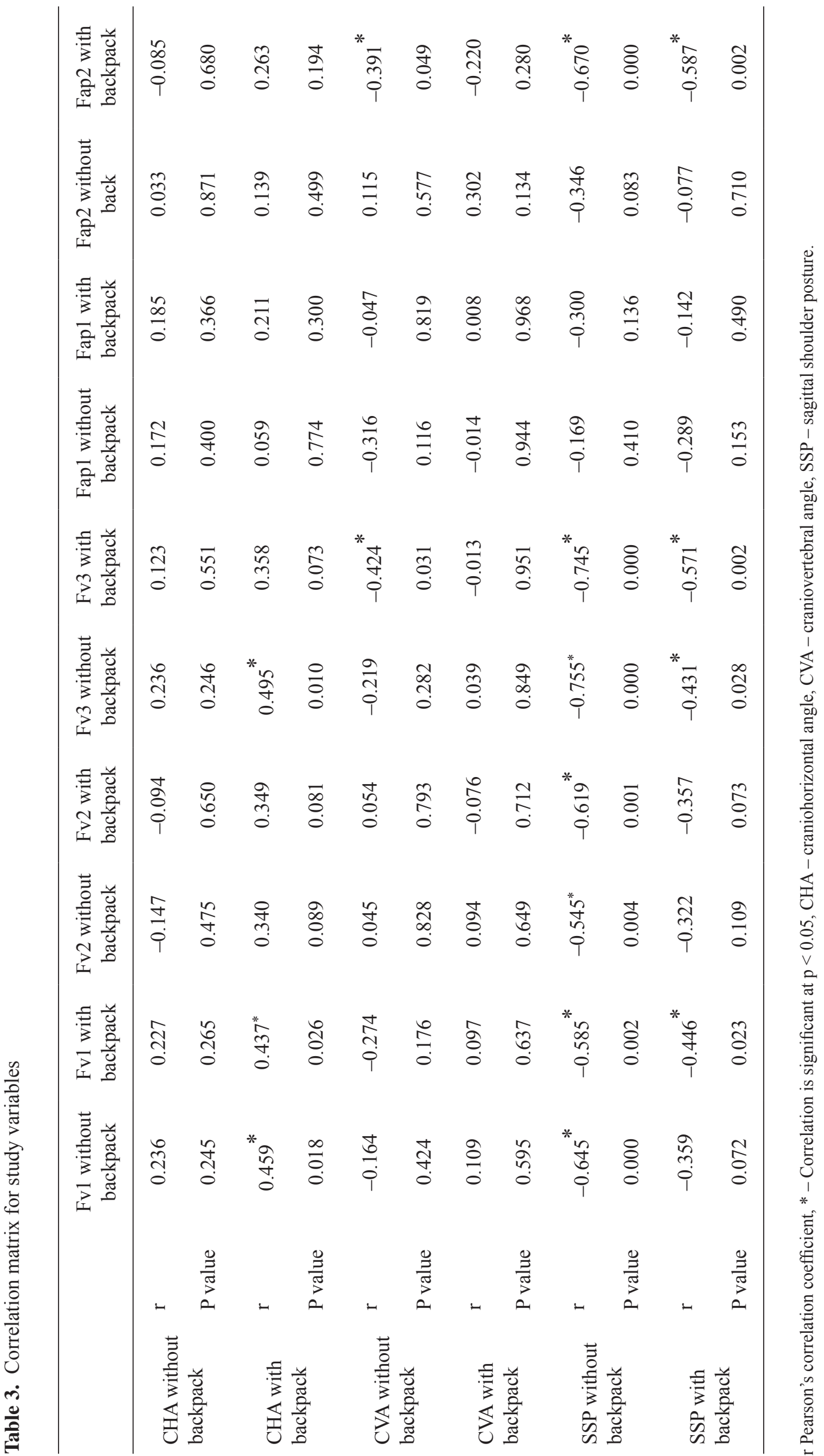


contact area) in school-age children (7-12 years), which goes against the current findings. The cause of disagreement may be the difference in the nature of the measured outcomes; the GRFs do not provide any information about where the forces are being applied on the foot [9], whereas the in-shoe plantar pressure systems allow quantification of the amount of vertical GRF being applied on each region of the plantar surface of the foot [11].

However, Castro et al. [8] found greater normalized anterior-posterior and vertical GRFs and greater plantar pressure peaks in the rearfoot, forefoot and hallux when adult participants walked carrying a backpack at high gait cadences compared to walking at low gait cadences. These measures were lower during normal walking compared to low and high gait cadence while carrying a backpack. Hence, the participants of the current study were asked to adopt their walk speed during the measurement procedure.

Chow et al. [15] attributed the increase in the vertical GRF to the changes in gait pattern during backpack carriage: The child leans the head and trunk forward, which moves the body center of gravity forward and so increases the acceleration, which creates a higher force during the heel strike phase, when the body segmental speeds require the highest inertial forces to decelerate the human body and backpack masses. On the other hand, during the propulsion phase of the stance phase, a more intense force is needed to accelerate the system $[5,46]$. These findings are supported by the significant increase in the normalized anterior-posterior GRFs: anterior braking and posterior propulsive forces during backpack carriage. By extension, joint contact forces will also be increased, particularly if muscle activity cannot overcome the increased load [42]. So, the participants were assessed during their comfortable gait to control the effects of the walking pattern.

However, Castro et al. [10] found greater normalized anterior-posterior and vertical GRFs (impact and thrust maximum) when the participants walked carrying a backpack at high cadences compared to walking with low cadences. Hence, testing the participants during their comfortable walking speed may be the cause of the nonsignificant increase in the normalized vertical GRFs. Meanwhile, there was agreement in the anterior-posterior GRFs. So, the low cadence can be considered a protective gait pattern during load carriage, aiming to minimize the possible harmful effects of the high vertical GRFs over the musculoskeletal system.

There was an increase in the magnitudes of the anterior braking force during backpack carriage compared to the unloaded condition that is confirmed by the findings of Castro et al. [8], who reported that the shear forces increased more than did the proportion of the load (normalized values). In addition, the increased shear force is supported by the recent findings [30], which reported that carrying a backpack disturbs the anterior-posterior stability more than no load. Moreover, carrying a backpack increases the area of the centre of pressure displacement in the anterior-posterior direction [35], which may explain the increased values of anterior-posterior forces (Fap1 and Fap2) during backpack carriage.

The current study revealed that there is moderate positive relation between the CHA and the normalized Fv1, which means that increasing the CHA with more upper cervical extension will increase the braking force of the vertical GRF, which was supported by Majumdar et al. [26] who reported that the forward head lean and vertical GRF increased significantly during carrying a military backpack. On the other hand, they found that the changes in anterior-posterior GRF were not significant in any loading condition and did not show any linearity or proportionality, which was against the findings of the present study. The participants' age may be the cause of the contradiction. However, some studies [25-27] reported that increasing the backpack weight increases the vertical and anterior-posterior GRFs, which is consistent with the current findings regarding the anterior backing and posterior propulsive forces.

The negative correlation between SSP and posterior propulsive force can be explained by the observations of Castro et al. [8], who found that the medial forefoot presented the largest increase in the pressure when carrying a backpack while the smallest increases occurred in the lateral forefoot. They suggested that when walking with a backpack the toe region was more needed than when walking without a backpack. Thus, the forces required to advance the body from the mid-stance to toe-off were reduced as a consequence of the decrease in the passive movement of the body [4]. Moreover, during backpack carriage, the load was carried more on the forefoot than the heel [7], which may explain why there was a negative correlation between the SSP and the posterior propulsive force without a correlation with the anterior braking force, which could be considered as a safe strategy by moving the center of mass forward.

\section{Limitations}

The study had some limitations. First, the comfortable walking speed was chosen to prevent disturbances in the gait pattern and ensure normal walking [11], which was considered a more realistic condition. Second, the mediallateral GRF was not measured because its values represented a very small percentage of the children's BW, as the children's BW is low. Third, the barefoot assessment of children was adopted to avoid the effect of different shoe types that may affect the head posture and GRFs. Finally, only the GRFs of the right foot had been measured depending on the assumption of gait symmetry [19], and a similar GRF between the right and left foot [40], which 
minimizes the effect of this limitation. Therefore, further study should examine the effect of prolonged walking with backpacks on the relationship between the neck angles and GRFs. More studies evaluating the effects of the different footwear on neck angles and GRFs during backpack carriage should be conducted to examine its effect on force absorption and posture alignment.

\section{Conclusion}

Carrying a backpack weighing $15 \%$ of the child's BW changes the head posture and increases the normalized value of anterior-posterior shear force compared to the unloaded condition. Moreover, decreasing the SSP is associated with increasing the load acceptance, thrusting and posterior propulsive forces. So, it is recommended to give advice to parents about the ideal weight of a backpack and carrying style to reduce the risk of posture and balance disturbance.

Conflict of interest: Authors state no conflict of interest.

\section{References}

1. Ahmad H.N., Barbosa T.M. (2019) The effects of backpack carriage on gait kinematics and kinetics of schoolchildren. Sci. Rep., 9: 3364. DOI: 10.1038/s41598-01940076-w.

2. Alghadier M. (2015) The effect of backpack weight on dynamic foot-loading patterns in Saudi school boys aged 7 to 12 years. Physiotherapy, 101: e51-e52. DOI: 10.1016/j.physio.2015.03.169.

3. Birrell S.A., Haslam R.A. (2009) The effect of military load carriage on 3-D lower limb kinematics and spatiotemporal parameters. Ergonomics, 52: 1298-1304. DOI: 10.1080/00140130903003115.

4. Birrell S.A., Haslam R.A. (2010) The effect of load distribution within military load carriage systems on the kinetics of human gait. Applied Ergonomics, 41(4), 585-590. https://doi.org/10.1016/j.apergo.2009.12.004.

5. Birrell S.A., Hooper R.H., Haslam R.A. (2007) The effect of military load carriage on ground reaction forces. Gait and Posture, 26(4): 611-614. DOI: 10.1016/j. gaitpost.2006.12.008.

6. Brackley H.M., Stevenson J.M., Selinger J.C. (2009) Effect of backpack load placement on posture and spinal curvature in prepubescent children. Work, 23(3), 351360. https://doi.org/10.3233/WOR-2009-0833

7. Carlsöö S. (1964) Influence of frontal and dorsal loads on muscle activity and on the weight distribution in the feet. Acta Orthopaedica, 28: 1347-1362. DOI: $10.3109 / 17453676408989326$.
8. Castro M., Abreu S., Sousa H., Machado L., Santos R., Vilas-Boas J.P. (2013) Ground reaction forces and plantar pressure distribution during occasional loaded gait. Appl. Ergon., 44(3): 503-509. DOI: 10.1016/j. apergo.2012.10.016.

9. Castro M.P.D., Meucci M., Soares D.P., Fonseca P., Borgonovo-Santos M., Sousa F., Vilas-Boas J.P. (2014) Accuracy and repeatability of the gait analysis by the walkinsense system. BioMed Res. Int., 11. DOI: $10.1155 / 2014 / 348659$.

10. Castro M.P., Figueiredo M.C., Abreu S., Sousa H., Machado L., Santos R., Vilas-Boas J.P. (2015) The influence of gait cadence on the ground reaction forces and plantar pressures during load carriage of young adults. Appl. Ergon., 49: 41-46. DOI: 10.1016/j.apergo.2015.01.004.

11. Cavanagh P.R., Ulbrecht J.S. (1994) Clinical plantar pressure measurement in diabetes: rationale and methodology. The Foot, 4: 123-135. DOI: 10.1016/0958-2592(94)90017-5.

12. Chang W.R., Chang C.C., Matz S. (2011) The effect of transverse shear force on the required coefficient of friction for level walking. Human Factors, 53: 461-473. DOI: $10.1177 / 0018720811414885$

13. Chansirinukor W., Wilson D., Grimmer K., Dansie B. (2001) Effects of backpacks on students: Measurement of cervical and shoulder posture. Aus. J. Physiother., 47(2): 110-116. DOI: 10.1016/S0004-9514(14)60302-0.

14. Chen Y.L., Mu Y.C. (2018) Effects of backpack load and position on body strains in male schoolchildren while walking. PLoS ONE. DOI: 10.1371/journal. pone. 0193648 .

15. Chow D.H.K., Kwok M.L.Y., Au-Yang A.C.K., Holmes A.D., Cheng J.C.Y., Yao F.Y.D., Wong M.S. (2006) The effect of load carriage on the gait of girls with adolescent idiopathic scoliosis and normal controls. Med. Eng. Phys., 28(5): 430-437. DOI: 10.1016/j. medengphy.2005.07.013.

16. Cole T.J., Bellizzi M.C., Flegal K.M., Dietz W.H. (2000) Establishing a standard definition for child overweight and obesity worldwide: international survey. BMJ, 320(7244): 1240-1243. DOI: 10.1136/bmj.320.7244.1240.

17. Drzał-Grabiec J., Truszczyńska A., Rykała J., Rachwał M., Snela S., Podgórska J. (2015) Effect of asymmetrical backpack load on spinal curvature in school children. Work, 51(2): 383-388. DOI: 10.3233/WOR-141981.

18. Farzaneh B., Hassan S., Fadilah T. (2009) The effect of backpacking load carriage on ground reaction forces in children. In: International Conference on Educational Research and Practice (ICERP), Marriot Hotel, Putrajaya, (p. 32).

19. Goble D.J., Marino G.W., Potvin J.R. (2003) The influence of horizontal velocity on interlimb symmetry in normal walking. Hum. Mov. Sci., 22: 271-283. DOI: 10.1016/ S0167-9457(03)00047-2. 
20. Hadžiomerović A.M., Jaganjac A., Avdic D., Pašalić A., Kaljić E., Domljan D., Omerović E. (2018) School bags and associated back pain. J. Health Sci., 8(1): 10-19.

21. Janakiraman B., Ravichandran H., Demeke S., Fasika S. (2017) Reported influences of backpack loads on postural deviation among school children: A systematic review. J. Educ. Health Promot., 6. DOI: 10.4103/jehp. jehp_26_15.

22. Johnson R.F., Knapik J.J., Merullo D.J. (1995) Symptoms during load carrying: effects of mass and load distribution during a 20-km road march. Percept. Mot. Skills, 81: 331-338. DOI: 10.2466/pms.1995.81.1.331.

23. Jorge J.G., de Faria A.N., Furtado D.A., Pereira A.A., de Carvalho E.M., Dionísio V.C. (2018) Kinematic and electromyographic analysis of school children gait with and without load in the backpack. Res. Biomed. Eng., 34(1): 9-18. DOI: 10.1590/2446-4740.04317.

24. Li S.S.W., Chow D.H.K. (2018) Effects of backpack load on critical changes of trunk muscle activation and lumbar spine loading during walking. Ergonomics, 6(4): 553-565. DOI: 10.1080/00140139.2017.1365950.

25. Lloyd R., Cooke C.B. (2000) Kinetic changes associated with load carriage using two rucksack designs. Ergonomics, 43(9): 1331-1341. DOI: 10.1080/001401300421770.

26. Majumdar D., Pal M.S., Majumdar D. (2010) Effects of military load carriage on kinematics of gait. Ergonomics, 53(6): 782-791. DOI: 10.1080/00140131003672015.

27. Majumdar D., Pal M.S., Pramanik A., Majumdar D. (2013) Kinetic changes in gait during low magnitude military load carriage. Ergonomics, 56(12): 1917-1927. DOI: 10.1080/00140139.2013.835871.

28. Mo S.W., Xu D.Q., Li J.X., Liu M. (2013) Effect of backpack load on the head, cervical spine and shoulder postures in children during gait termination. Ergonomics, 56(12): 1908-1916. DOI: 10.1080/00140139.2013.851281.

29. Mosaad D.M., Abdel-Aziem A.A. (2015) Backpack carriage effect on head posture and ground reaction forces in school children. Work, 52(1). DOI: 10.3233/WOR152043 .

30. Mosaad D.M., Abdel-Aziem A.A. (2018) Postural balance and neck angle changes in school children while carrying a traditional backpack versus a double-sided bag. Biomed. Hum. Kinet., DOI: 10.1515/bhk-2018-0010.

31. Pau M., Leban B., Corona F., Gioi S., Nussbaum M.A. (2016) School-based screening of plantar pressures during level walking with a backpack among overweight and obese schoolchildren. Ergonomics, 59(5): 697-703. DOI: 10.1080/00140139.2015.1077275.

32. Peduzzi de Castro, M., Abreu S., Pinto V., Santos R., Machado L., Vaz M., Vilas-Boas J.P. (2014) Influence of pressure-relief insoles developed for loaded gait (backpackers and obese people) on plantar pressure distribution and ground reaction forces. Appl. Ergon., 45: 10281034. DOI: 10.1016/j.apergo.2014.01.005.
33. Perrone M., Orr R., Hing W., Milne N., Pope R. (2018) The impact of backpack loads on school children: A critical narrative review. Int. J. Environ. Res. Public Health, 15(2529). DOI: 10.3390/ijerph15112529.

34. Piscoya J.L., Fermor B., Kraus V.B., Stabler T.V., Guilak F. (2005) The influence of mechanical compression on the induction of osteoarthritis-related biomarkers in articular cartilage explants. Osteoarthritis and Cartilage, 13(2): 1092-1099. DOI: 10.1016/j.joca.2005.07.003.

35. Politti F., Gonzalez T. de O., Gomes C.A.F. de P., el Hage Y., Amaral A.P., Amorim C.F., Biasotto-Gonzalez D.A. (2012) Effect of the usual weight of a backpack on body sway during quiet standing. J. Phys. Ther. Sci., 24: 1079-1082. DOI: 10.1589/jpts.24.1079.

36. Poursadeghiyan M., Azrah K., Biglari H., Ebrahimi M.H., Yarmohammadi H., Baneshi M.M., Khammar A. (2017) The effects of the manner of carrying the bags on musculoskeletal symptoms in school students in the city of Ilam, Iran. Ann. Trop. Med. Public Health, 10(3). DOI: 10.4103/ATMPH.ATMPH 10917.

37. Rai A., Agarwal S., Bharti S. (2013) Postural effect of back packs on school children: its consequences on their body posture. Int. J. Health Sci. Res., 3(10): 109-116.

38. Raine S., Twomey L.T. (1997) Head and shoulder posture variations in 160 asymptomatic women and men. Arch. Phys. Med. Rehabil., 78: 1215-1223. DOI: 10.1016/ S0003-9993(97)90335-X.

39. Schuh-Renner A., Grier T.L., Canham-Chervak M., Hauschild V.D., Roy T.C., Fletcher J., Jones B.H. (2017) Risk factors for injury associated with low, moderate, and high mileage road marching in a U.S. Army infantry brigade. J. Sci. Med. Sport, 20(Suppl 4): S28-S33. DOI: 10.1016/j.jsams.2017.07.027.

40. Seeley M.K., Umberger B.R., Shapiro R. (2008) A test of the functional asymmetry hypothesis in walking. Gait and Posture, 28: 24-28. DOI: 10.1016/j.gaitpost.2007.09.006.

41. Sharan D., Mohandoss M., Ranganathan R., Makkuva clude A., Kavoor J.J. (2015) A systematic review of risk factors for musculoskeletal pain due to heavy backpacks in school children. In: Proceedings 19th Triennial Congress of the IEA, Melbourne 9-14 August 2015 (p. 14).

42. Shasmin H.N., Abu Osman N.A., Razali R., Usman J., (2007) The effect of load carriage among primary school boys: A preliminary study. J. Mech. Med. Biol., 7(3): 265-274. DOI: 10.1142/s0219519407002285.

43. Simpson K.M., Munro B.J., Steele J.R. (2012) Effects of prolonged load carriage on ground reaction forces, lower limb kinematics and spatio-temporal parameters in female recreational hikers. Ergonomics, 55(3), 316-326. DOI: $10.1080 / 00140139.2011 .642004$.

44. Singh T., Koh M. (2009) Effects of backpack load position on spatiotemporal parameters and trunk forward lean. Gait and Posture, 29: 49-53. DOI: 10.1016/j. gaitpost.2008.06.006. 
45. Skaggs D.L., Early S.D., D’Ambra P., Tolo V.T., Kay R.M. (2006) Back pain and backpacks in school children. J. Pediatr. Orthop., 26(3): 358-363. DOI: 10.1097/01. bpo.0000217723.14631.6e

46. Tilbury-Davis D.C., Hooper R.H. (1999) The kinetic and kinematic effects of increasing load carriage upon the lower limb. Hum. Mov. Sci., 18(5): 693-700. DOI: 10.1016/S0167-9457(99)00026-3.

47. Twomey L. (1994) Posture of the head, shoulders and thoracic spine in comfortable erect standing. Aus. J. Physiother, 40: 25-32. DOI: 10.1016/S0004-9514(14)60451-7.

48. Vaghela N., Parekh S., Padsala D., Patel D. (2019) Effect of backpack loading on cervical and sagittal shoulder posture in standing and after dynamic activity in school going children. J. Family Med. Prim. Care, 8(3): 1076-1081. DOI: 10.4103/jfmpc.jfmpc_367_18.
49. Zaidi M., Ansari F., Waseem H., Fahim S., (2016) Association of musculoskeletal pain with heavy bagpacks among school children. In: Proc. 14 th International Conference on Statistical Sciences Karachi, Pakistan March 14-16, Vol 29, (pp. 229-238).

\section{Received 23.08.2019 \\ Accepted 08.01.2020}

(C) University of Physical Education, Warsaw, Poland

Acknowledgments

The authors wish to acknowledge their gratitude to the children, and their parents. 